\title{
Evolving views and practices of antiretroviral treatment prescribers in Australia
}

\author{
Early ART \\ initiation was \\ supported \\ by most \\ prescribers \\ primarily as \\ treatment
}

rather than as

prevention

Philippe C G Adam

Susan Kippax

BA(Hons), PhD'

Levinia Crooks

BA(Hons), DipEd, MBA 2,3 $^{2}$

Jeffrey J Post MBBS(Hons), PhD, FRACP ${ }^{1,4}$

Michael R Kidd

AM, MD, FRACGP

Sean Slavin

PhD 1.6

Edwina J Wright MBBS, PhD, FRACP?

John B F de Wit MSc, PhD

1 University of New South Wales,

Sydney, NSW.

2 Australasian Society for HIV Medicine Sydney, NSW.

3 La Trobe University, Melbourne, VIC.

4 Prince of Wales Hospital, Sydney, NSW.

5 Flinders University, Adelaide, SA.

6 Australian Federation of

AIDS Organisations

Sydney, NSW.

7 The Alfred,

Melbourne, VIC.

limin.mao@unsw.edu.au

doi: 10.5694/mjal4.00443
$\mathrm{T}$ he most recent Australian HIV clinical treatment guidelines support early antiretroviral treatment (ART) initiation, whereby asymptomatic patients should start treatment once their CD4+ cell count approaches 500 cells $/ \mathrm{mm}^{3}$ or even earlier. ${ }^{1}$ This is consistent with a global movement based on emerging evidence and growing expert consensus that early initiation of ART has clear clinical benefits to individual patients and a potentially important population effect in reducing HIV transmission to patients' uninfected sexual partners., ${ }^{2,3}$ Compared with earlier regimens, current ART regimens are less toxic, have simpler dosing requirements and lower pill burdens, and do not generally require as high a degree of drug adherence to achieve viral suppression. ${ }^{3}$ These developments have influenced policy regulation, resource allocation and ART initiation practices in Australia $a^{4,5}$ and other high-income countries. ${ }^{6,7}$

There is a paucity of research on prescriber characteristics relating to ART initiation practices. In some of the few studies that have been conducted, prescribers' knowledge about ART guideline changes, familiarity with ART regimens, experience of and specialty in treating HIV-positive patients, and beliefs in ART effectiveness were closely associated with their initiation practices. ${ }^{7-9}$

We examined whether there have been recent changes in Australian ART prescribers' perceptions of and practices towards early ART initiation. We also assessed prescriber factors that are likely to influence recommendation of ART initiation, including prescribers' perceptions of their HIV-positive patients.

\section{Methods}

Repeated online cross-sectional surveys targeting all ART prescribers in Australia were conducted in 2012 (mid April to mid May) and 2013 (mid

\section{Abstract}

Objective: To examine whether there have been recent changes in Australian antiretroviral treatment (ART) prescribers' perceptions and practices relating to early ART initiation, which was defined as commencing ART when a patient's CD4+ T-cell count approaches 500 cells $/ \mathrm{mm}^{3}$ or immediately after a patient is diagnosed with HIV.

Design, participants and setting: Self-completed, anonymous, crosssectional surveys, targeting all ART prescribers in Australia, were conducted online in 2012 and 2013. The surveys included questions on prescriber factors, CD4+ T-cell count at which prescribers would most strongly recommend ART initiation, and perceived patient characteristics that could change prescribers' practices of early initiation of ART.

Main outcome measures: Proportions of ART prescribers recommending early ART initiation.

Results: We analysed responses from 108 participants in 2012 and 82 participants in 2013. In both years, more male than female prescribers participated. The median age of participants was 49 years in 2012 and 50 years in 2013. In both rounds, over $60 \%$ had more than 10 years' experience in treating HIV-positive patients. More prescribers in 2013 stated that they would most strongly recommend early ART initiation compared with those in 2012 (50.0\% [95\% Cl, 38.7\%-61.3\%] v $26.9 \%$ [95\% Cl, $18.8 \%-36.2 \%] ; P=0.001)$. The prescribers' primary concern was more about individual patient than public health benefit. Out of 824 patients for whom ART was initiated, as reported by prescribers in 2013, only 108 (13.1\% [95\% Cl, 10.9\%-15.6\%]) were given ART primarily to prevent onward HIV transmission. The number of patients for whom ART was initiated was significantly associated with prescribers' HIV caseload even after adjusting for prescriber type (adjusted odds ratio, 1.73 [95\% Cl, 1.47-2.03]; $P<0.001$ ); of the 37 who had initiated ART for 10 or more patients, 29 had a high HIV caseload. In 2013, 60 prescribers (73.2\% [95\% CI, 62.2\%-82.4\%]) reported that they routinely recommended ART to treatment-naive, asymptomatic patients with a CD4+ T-cell count of 350-500 cells $/ \mathrm{mm}^{3}$.

Conclusion: Our findings show increasing acceptance of and support for early ART initiation primarily as treatment and not as prevention.

May to mid July). At each round, invitations were sent to online registrants of the Australasian Society for HIV Medicine (ASHM), which provides ongoing professional training for clinicians throughout Australia who specialise in treating patients with HIV. The surveys were self-completed and anonymous, and participants did not receive reimbursement for completing them. Detailed descriptions of the 2012 round are published elsewhere. ${ }^{10}$ The study was approved by the Human Research Ethics Advisory Panel I of the University of New South Wales (which reviews social and health research).

In both rounds, the surveys included questions on demographics, clinical experience in treating HIV-positive patients and primary concerns relating to recommendation of ART initiation. Participants were also asked to indicate the CD4+ T-cell count at which they would most strongly recommend ART initiation. Answers to questions on prescribers' primary concerns when recommending ART initiation - one in relation to ART's health benefits to individual patients and the other in relation to its benefits to population health - were measured by 11-point Likert scales, ranging from completely disagree (0) to completely agree (10).

In 2013, new questions were added to assess prescribers' beliefs in and practices of early initiation of ART. Early initiation was defined as commencing ART when a patient's CD4+ 
T-cell count approaches 500 cells/ $\mathrm{mm}^{3}$ or immediately after a patient is diagnosed with HIV irrespective of CD4+ T-cell count. A series of scenario-based questions was also added, to explore perceived patient characteristics that could potentially change prescribers' practices of recommending early initiation of ART. The scenarios examined a range of patient characteristics, including demographic factors (eight items; eg, unstable housing), behavioural factors (six items; eg, male-to-male sex) and clinical factors (eight items; eg, an HIV diagnosis in the past 6 months). To reduce response bias, each new item was presented in a random order, accompanied by a five-point Likert scale, ranging from strongly disagree (1) to strongly agree (5).

Prescribers who were treating no more than $10 \mathrm{HIV}$-positive patients were classified as having a low HIV caseload; those treating 11-50 as having a medium HIV caseload; and those treating more than 50 as having a high HIV caseload.

Descriptive statistics, including estimated confidence intervals (CIs), are reported. Data from the two rounds were compared using $\chi^{2}$ tests. Adjusted odds ratios (AORs) and $95 \%$ CIs from multivariable generalised linear regression analyses for the ordinal dependent variables are reported for factors significantly associated with prescribers' attitudes towards and practices of ART initiation. All data analyses were performed in STATA 11.2 (StataCorp).

\section{Results}

We analysed 108 valid responses (out of 113 eligible ones) from 2012 and 82 valid responses (out of 91 eligible ones) from 2013. The estimated response rates were $51 \%$ in 2012 (113/222) and $41 \%$ in 2013 (91/222); about 200-250 ART prescribers had been active ASHM online registrants over the survey periods.

Prescribers' demographic and clinical profiles were very similar in the two rounds (Box). In both rounds, more than half of the respondents were male. The median age of participants was 49 years in 2012 and

Demographic characteristics and clinical experience of antiretroviral treatment prescribers who participated in surveys in 2012 and 2013

\begin{tabular}{|c|c|c|c|}
\hline & $2012(n=108)$ & $2013(n=82)$ & $P$ \\
\hline \multicolumn{4}{|l|}{ Demographics } \\
\hline Gender & & & 0.79 \\
\hline Male & $61(56.5 \%)$ & $50(61.0 \%)$ & \\
\hline Female & $46(42.6 \%)$ & $31(37.8 \%)$ & \\
\hline Transgender & $1(0.9 \%)$ & $1(1.2 \%)$ & \\
\hline Age & & & 0.84 \\
\hline$<45$ years old & $38(35.2 \%)$ & $26(31.7 \%)$ & \\
\hline 45-54 years old & $42(38.9 \%)$ & $32(39.0 \%)$ & \\
\hline$\geqslant 55$ years old & $28(25.9 \%)$ & $24(29.3 \%)$ & \\
\hline \multicolumn{4}{|l|}{ Clinical experience } \\
\hline Primary work place location & & & 0.52 \\
\hline New South Wales & $52(48.1 \%)$ & $46(56.1 \%)$ & \\
\hline Victoria & $27(25.0 \%)$ & $16(19.5 \%)$ & \\
\hline Other Australian states or territories & $29(26.9 \%)$ & $20(24.4 \%)$ & \\
\hline Prescriber type & & & 0.006 \\
\hline Hospital-based infectious diseases specialist & $23(21.3 \%)$ & $9(11.0 \%)$ & \\
\hline Sexual health centre-based physician & $28(25.9 \%)$ & $35(42.7 \%)$ & \\
\hline Section 100 accredited general practitioner & $44(40.7 \%)$ & $36(43.9 \%)$ & \\
\hline Other* & $13(12.0 \%)$ & $2(2.4 \%)$ & \\
\hline Period of treating HIV-positive patients & & & 0.51 \\
\hline $1-5$ years & $23(21.3 \%)$ & $14(17.1 \%)$ & \\
\hline $6-10$ years & $18(16.7 \%)$ & $13(15.9 \%)$ & \\
\hline$>10$ years & $65(60.2 \%)$ & $55(67.1 \%)$ & \\
\hline Missing & $2(1.9 \%)$ & 0 & \\
\hline $\begin{array}{l}\text { Number of HIV-positive patients as a primary } \\
\text { provider }\end{array}$ & & & 0.15 \\
\hline 1-10 patients (low HIV caseload) & $22(20.4 \%)$ & $9(11.0 \%)$ & \\
\hline 11-50 patients (medium HIV caseload) & $43(39.8 \%)$ & $33(40.2 \%)$ & \\
\hline > 50 patients (high HIV caseload) & $41(38.0 \%)$ & $40(48.8 \%)$ & \\
\hline Missing & $2(1.9 \%)$ & 0 & \\
\hline
\end{tabular}

*In 2012, other included five hospital-based prescribers of unspecified specialty, five hospital-based immunology specialists, one prison-based physician, one sexual health physician working in private practice and one participant for whom prescriber type was missing; in 2013, other included one non-hospital-based immunology specialist and one GP who did not have Section 100 accreditation.

50 years in 2013. Each year, close to half were practising in New South Wales, over $60 \%$ had more than 10 years' experience in treating HIVpositive patients, and about $40 \%$ had a high HIV caseload. The sample in 2013 had significantly fewer hospitalbased infectious diseases specialists than that in $2012(P=0.006)$.

\section{Support for early ART initiation}

In 2013, 41 prescribers indicated that they would most strongly recommend early ART initiation compared with 29 prescribers in 2012 (50.0\%
[95\% CI, $38.7 \%-61.3 \%$ ] v $26.9 \%$ [95\% CI, $18.8 \%-36.2 \%] ; P=0.001)$. This difference remained significant after adjustment for prescriber type (AOR, 1.78 [95\% CI, 1.21-2.62]; $P=0.003$ ).

However, only 16 prescribers in 2013 $(19.5 \%$ [ $95 \%$ CI, $11.6 \%-29.7 \%])$ would both most strongly recommend and routinely recommend early ART initiation. This was despite the fact that many agreed that available evidence supports early ART initiation. In 2013, 43 prescribers $(52.4 \%$ [95\% CI, $41.1 \%-$ 63.6\%]) agreed that ART initiation at a CD4+ T-cell count approaching 
500 cells $/ \mathrm{mm}^{3}$ was supported by evidence, and 23 prescribers (28.0\% [95\% CI, $18.7 \%-39.1 \%]$ ) agreed that immediate ART initiation after a diagnosis of HIV, irrespective of CD4+ T-cell count, was supported by evidence.

In 2013, 82 prescribers reported that they had initiated ART for a total of 824 HIV-positive patients in the previous 12 months - an average of 10 patients per year per prescriber (median, 6; range, 0-60). The number of patients for whom ART was initiated was significantly associated with prescribers' HIV caseload even after adjusting for prescriber type (AOR, 1.73 [95\% CI, 1.47-2.03]; $P<0.001)$; of the 37 who had initiated ART for 10 or more patients, 29 had a high HIV caseload.

\section{ART primarily as treatment, not as prevention}

In both rounds, prescribers' primary concern was predominantly individual patient benefit (102 prescribers [94.4\%] in $2012 \mathrm{v} 80$ prescribers [97.6\%] in 2013; $P=0.29$ ). A small proportion indicated any population benefit as a primary concern (20 prescribers [18.5\%] in $2012 \mathrm{v} 23$ prescribers [28.0\%] in 2013; $P=0.12$ ).

According to prescribers in 2013, only a small proportion of the 824 patients for whom ART was initiated (108 patients [13.1\%; 95\% CI, $10.9 \%-$ $15.6 \%$ ]) were given ART to prevent onward HIV transmission. ART initiation primarily for HIV prevention was also associated with prescribers' HIV caseload, even after adjustment for prescriber type (AOR, 1.69 [95\% CI, 1.18-2.41]; $P=0.004) ; 27$ of 38 prescribers who had done so had a high HIV caseload.

\section{Conditional support for ART as prevention}

In 2013,60 prescribers $(73.2 \%$ [95\% CI, $62.2 \%-82.4 \%]$ ) reported that they routinely recommended ART to treatment-naive, asymptomatic patients with a CD4+ T-cell count of
$350-500$ cells $/ \mathrm{mm}^{3}$, as suggested by the latest clinical guidelines. ${ }^{1}$

However, a range of perceived patient characteristics were found to substantially alter the likelihood of ART recommendation by prescribers. Notably, all prescribers in 2013 stated that they would recommend ART to patients with an uninfected regular partner. Also, the vast majority would recommend ART to patients engaging in unprotected intercourse with any non-HIV-positive partner (80 prescribers [97.6\%; 95\% CI, 91.5\%-99.7\%]) or selling sex (79 prescribers [96.3\%; 95\% CI, 89.7\%-99.2\%]).

In contrast, ART was less likely to be recommended to patients with a history of medication non-adherence (34 prescribers [ $41.5 \%$; $95 \%$ CI, 30.7\%$52.9 \%]$ ) or a history of at least three missed clinical appointments in the previous 12 months (36 prescribers [43.9\%; 95\% CI, 33.0\%-55.3\%]).

\section{Discussion}

Australian ART prescribers have made swift yet cautious moves towards early ART initiation since 2012, keeping pace with clinical guideline changes. ${ }^{4,5,11}$ With regard to ART initiation, prescribers prioritise individual patient health over HIV prevention benefit. Early ART initiation was supported by most prescribers primarily as treatment rather than as prevention, with the exception of high HIV caseload prescribers.

While HIV caseload was found to be a key prescriber factor in early ART initiation, perceived patient characteristics (history of medication non-adherence and missed clinical appointments) also influenced clinician compliance with guideline-recommended, routine ART initiation (ie, at a CD4+ T-cell count of $350-500$ cells $/ \mathrm{mm}^{3}$ ). Poor clinical engagement and potential ART nonadherence, which suggest increased risk of onward HIV transmission, have previously been shown to be linked with patients' suboptimal viral suppression. ${ }^{12}$

In support of early ART initiation, the Australian Pharmaceutical Benefits Advisory Committee recently removed one fundamental structural barrier by expanding the government subsidy for ART to patients who are asymptomatic and have a CD4+ T-cell count above 500 cells $/ \mathrm{mm}^{3}$. Future research should assess the influence of this change on ART prescribers' views and practices.

The main limitations of this study are the small sample sizes and moderate response rates, although response rates are difficult to calculate accurately because the total number of clinicians who are able to prescribe ART in Australia is unknown. Given that there are about 1200 new HIV diagnoses annually in Australia ${ }^{13}$ and that ART was initiated for 824 patients in 2013, we probably captured a considerable proportion of ART prescribers. Finally, the repeated, anonymous, cross-sectional design precludes causal inferences and further analysis of changes for each individual prescriber.

Our findings show increasing acceptance of and support for early ART initiation primarily as treatment and not as prevention.

Acknowledgements: This study was funded by the National Health and Medical Research Council (NHMRC) (APP1021790). Limin Mao, Philippe Adam and John de Wit work at the Centre for Social Research in Health at the University of New South Wales, which is supported by the Australian Government Department of Health. We acknowledge our partner organisations (the Kirby Institute, the National Association of People with HIV Australia, the Australasian Society for HIV Medicine, the Australian Federation of AIDS Organisations, NSW Positive Central and Heterosexual HIV Service, Positive Life NSW and NSW Health), which are members of the research advisory committee that set the research agenda, provided advice, monitored the research progress and helped disseminate the research findings. We are grateful to the contribution made by the study participants. The NHMRC does not influence how research findings are reported, including in this article, and we had full access to all the survey data.

Competing interests: No relevant disclosures.

Received 26 Mar 2014, accepted 27 Aug 2014.

References are available online at www.mja.com.au. 
1 Australasian Society for HIV Medicine. Antiretroviral guidelines. US DHHS guidelines with Australian commentary. ASHM, 2013. http://arv. ashm.org.au (accessed Aug 2014).

2 Cohen MS, Chen YQ, McCauley M, et al. Prevention of HIV-1 infection with early antiretroviral therapy. NEngl J Med 2011; 365: 493-505.

3 Cohen MS, Gay CL. Treatment to prevent transmission of HIV-l. Clin Infect Dis 2010; 50 Suppl 3: S85-S95.

4 Kelly MD, Broom JK, Young S. The use of key performance indicators to benchmark individual clinic performance in managing a complex chronic infectious disease [letter]. Med J Aust 2012; 197: 330-331.

5 Smith D, Goggin L, Meldrum H, et al. Capturing the paradigm shift in HIV treatment: changing attitudes in the choice of combination antiretroviral drugs by high HIV caseload Australian GPs (1996-1997). AIDS Care 2000; 12: 41-47.

6 Krastinova E, Seng R, Yeni P, et al. Is clinical practice concordant with the changes in guidelines for antiretroviral therapy initiation during primary and chronic HIV-l infection? The ANRS PRIMO and COPANA cohorts. PLOS One 2013; 8: e71473.

7 Kurth AE, Mayer K, Beauchamp G, et al. Clinician practices and attitudes regarding early antiretroviral therapy in the United States. J Acquir Immune Defic Syndr 2012; 61: e65-e69.
8 Bogart LM, Kelly JA, Catz SL, Sosman JM. Impact of medical and nonmedical factors on physician decision making for HIV/AIDS antiretroviral treatment. J Acquir Immune Defic Syndr 2000; 23: 396-404.

9 Obadia Y, Souville M, Morin M, Moatti JP. French general practitioners' attitudes toward therapeutic advances in HIV care: results of a national survey. Int J STD AIDS 1999; 10: 243-249.

10 Mao L, de Wit J, Adam P, et al. Australian prescribers' perspectives on ART initiation in the era of "treatment as prevention". AIDS Care 2013; 25: 1375-1379.

1 Bloch M, Hoy J, Cunningham N, et al. Adherence to HIV treatment guidelines for comorbid disease assessment and initiation of antiretroviral therapy. $J$ Acquir Immune Defic Syndr 2012; 59 : 478-488.

12 Nordqvist O, Södergård B, Tully MP, et al. Assessing and achieving readiness to initiate HIV medication. Patient Educ Couns 2006; 62: 21-30.

13 The Kirby Institute. HIV, viral hepatitis and sexually transmissible infections in Australia. Annual surveillance report 2013. Sydney: The Kirby Institute, University of New South Wales, 2013. http://kirby.unsw.edu. au/sites/default/files/hiv/resources /2013AnnualSurvReport.pdf (accessed Aug 2014). 